\title{
A method for estimating the dynamical age of FR II-type radio sources from multi-frequency data
}

\author{
J. Machalski ${ }^{1}$, K. T. Chyży ${ }^{1}$, Ł. Stawarz $^{1,2}$, and D. Kozieł ${ }^{1}$ \\ 1 Astronomical Observatory, Jagellonian University, ul. Orla 171, 30244 Cracow, Poland \\ 2 Landessternwarte Heidelberg, Königstuhl, and MPI für Kernphysik, Saupfercheckweg 1, 69117 Heidelberg, Germany \\ e-mail: machalsk@oa.uj.edu.pl
}

Received 27 July 2006 / Accepted 21 September 2006

\begin{abstract}
Context. Determining the ages of powerful radio sources is crucial for understanding galaxy evolution, the activity cycle of galactic nuclei, and their influence on the surrounding intergalactic medium. So far, several different methods for estimating the age of classical double radio galaxies have been proposed and widely used in the literature, although each of them faces difficulty due to observational limitations and/or freedom in choosing the underlying model assumptions.

Aims. We propose a new approach to determining the ages of FR II type radio sources that, on one hand exploits a dynamical model developed for these objects by Kaiser et al. (1997, MNRAS, 292, 723) and, on the other hand, uses multifrequency radio observations not necessarily restricted to the high-resolution ones.

Methods. In particular, we applied the assumed dynamical model to a number of FR II type radio galaxies observed at different radio frequencies and fit - for each frequency separately - the model's free parameters to the quantities of the observed sources. Such a procedure, which in fact enlarged a number of observables, enabled us to determine relatively precise ages and other crucial characteristics (like the jets' kinetic power) for the analyzed sources.

Results. The resulting age estimates agree very well with those obtained with the "classical" spectral aging method for objects not older than $10 \mathrm{Myr}$, for which good-quality spectral data are available. However, this method is also applicable in the case of older sources than this and/or those for which the only available low-resolution radio data do not allow for detailed spectral aging studies. Interestingly, the estimated ages always correspond to the realistic values of the jets' advance velocity of $\sim 0.01-0.1 c$.

Conclusions. Our analysis indicates that the main factor precluding precise age determination for FR II type radio galaxies is related to the poorly known shape of the initial electron energy distribution injected by the jet's terminal shocks to the expanding lobes/cocoons. We briefly considered this issue and conclude that the broad-band single power-law form assumed here may be accurate enough for the age estimates, although most likely it does not strictly correspond to some well-defined realistic particle acceleration process. Instead, it should be considered as the simplest model approximation of the initial electron continuum, averaged over a very broad energy range and over the age of the source, with the effective spectral index that may be different for different sources, but within the relatively narrow range of $p=2.0-2.4$ suggested by our modeling.
\end{abstract}

Key words. galaxies: active - galaxies: evolution - radio continuum: galaxies

\section{Introduction}

The dynamical age of extragalactic radio sources is very important for first studying and then understanding details of the physical processes taking place in both active galactic nuclei (AGNs) which emit energy in the form of jets boring through the ambient intergalactic medium (IGM) and lobes or a cocoon consisting of shocked (backflowing) jet material and shocked IGM. The "standard model" for classical double radio sources (Blandford \& Rees 1974; Scheuer 1974) implies that the axial lengthening of the cocoon is governed by the balance between the jet's thrust and the ram pressure of the IGM, while the width of the cocoon is determined by its internal pressure. This scenario has formed the basis for all the later analytical models of the dynamics and radio-emission properties of powerful double radio sources (e.g., Begelman \& Cioffi 1989; Falle 1991; Nath 1995; Kaiser et al. 1997; Chyży 1997; Blundell et al. 1999; Manolakou \& Kirk 2002; Kino \& Kawakatu 2005).

All these dynamical models allow one to predict a number of observational parameters of the sources (e.g., linear size, width of the cocoon, radio power at different wavelengths) for their given ages and redshifts by assuming values of the models' basic free parameters (e.g., jet power, the external medium density and its gradient, etc.). A comparison of the predictions of the three main scenarios, i.e. those by Kaiser et al. (1997), Blundell et al. (1999), and Manolakou \& Kirk (2002), was recently undertaken by Barai \& Wiita (2006). They argued that no existing model could acceptably fit to the common properties of radio sources from the survey under consideration (3 CRR, 6CE, 7CRS), although the Manolakou \& Kirk model gave "better overall results than do either of the other two".

The age of a classical double radio source can be determined, in principle, from its apparent radio spectrum. There is no doubt that a radio continuum spectrum in different parts of a radio source should contain crucial information about its history, depending on the various energy losses (and gains) of the radiating particles. This simple idea, which relies on the determination of a single characteristic break frequency in the radio spectrum (at a given part of the source) caused by the above time-dependent losses, was used to estimate the radiative ages and expansion speeds in large samples of powerful 3CR sources (Myers \& Spangler 1985; Alexander \& Leahy 1987; 
Leahy et al. 1989; Carilli et al. 1991; Liu et al. 1992), as well as in samples of low-luminosity and intermediate-luminosity radio galaxies (Klein et al. 1995; Parma et al. 1999). However, the spectral break and steepening of the spectrum beyond this break need not be entirely due to radiative aging. A possible role of the local magnetic field structure and evolution, details of the backflow process of the cocoon material, or the difficulties in disentangling various energy losses of the radiating particles have been raised in a number of papers (e.g. Rudnick et al. 1994; Eilek \& Arendt 1996; Jones et al. 1999).

The task of resolving some of the above difficulties was undertaken by Kaiser (2000, hereafter referred to as K2000), who constructed a 3-dimensional model of the synchrotron emissivity for a cocoon of an FR II-type radio source and applied it to the self-similar model of the cocoon's dynamics developed by Kaiser \& Alexander (1997, hereafter referred to as KA) and its extension by Kaiser et al. (1997, hereafter KDA). In the K2000 model, the cocoon is split into small-volume elements whose surface-brightness evolution is traced individually. The author argues that the proposed careful analysis of the bulk backflow process and of the electron energy losses (both radiative and adiabatic) can accurately estimate the sources' spectral ages, in addition to agreement with the estimates of their dynamical ages. Blundell \& Rawlings (2000) contend however, that this could only be the case if they were much younger than $10 \mathrm{Myr}$.

Analyzing the K2000 model, we realized that in the majority of the extended FR II-type radio sources - even those without distorted lobe structures - the surface-brightness profiles are far from the expected smooth shapes, making the fitted free parameters highly uncertain. In addition, the K2000 method requires rather high-resolution observations of the radio lobes. But usually high-resolution observations of low-brightness sources cause a serious loss of the flux density, so that the K2000 method can in fact only be applied to a few of the strongest sources such as Cyg A.

The KDA model has been successively used by Machalski et al. $(2004 a, b)$ to derive the basic physical parameters of the FR II-type radio sources with known ages, namely the jet power, the central density of the ambient medium, the cocoon's energy density and pressure, and the total energy deposited in the radio source. All of these values were derived by means of fitting the model free parameters to the observed parameters of the source: its redshift, monochromatic radio luminosity at $1.4 \mathrm{GHz}$, projected linear size, and the cocoon's axial ratio. In this paper we propose a simplified method of determining the age of powerful radio sources that is based on a further exploitation of the KDA model, which avoids the limitations present in the K2000 method. In particular, we show that performing this fitting procedure for a $f e w$ observing frequencies, i.e. enlarging the number of the observables used by adding the radio spectrum data of the entire cocoon, a unique set of the age and values for the two fundamental model parameters - the jet power and the central density of the ambient medium - can be found. This, in turn, enables us to determine the age of the analyzed source relatively precisely, even if the available (low-resolution) radio data do not allow for detailed spectral aging studies.

The paper is organized as follows. First, a brief presentation of the analytical KDA model, together with its application to the dynamical aging studies, are given in Sect. 2. In Sect. 3 we present the dataset used in the subsequent analysis. Examples of the dynamical age determination for the radio galaxy Cygnus A and its dependence on the assumed model parameters are given in Sect. 4. A summary of how the method is applied to a variety of other FR II type radio sources is presented in Sect. 5.
Remarks on the implications of the results for modeling the electron injection-spectrum in lobes of powerful radio sources are given in Sect. 6. Final conclusions are presented in Sect. 7.

\section{The KDA model}

In this section the model application is described more in details.

\subsection{Source dynamics}

The dynamical expansion of an FR II-type radio source built in the KDA model comes from the dynamics described in the selfsimilar KA model. Based on the original idea of Blandford \& Rees (1974) and Scheuer (1974), it assumes that the classical double radio structure is formed by twin jets emerging from the AGN in opposite directions into a surrounding tenuous environment. The jets terminate in strong shocks where the jet particles are shocked, accelerated, and finally inflate the cocoon. A density distribution of the unperturbed external gas is approximated by a power-law radial distribution scaling with distance $r>a_{0}$ from the center of the host galaxy as

$\rho(r)=\rho_{0}\left(\frac{r}{a_{0}}\right)^{-\beta}$

where $\rho_{0}$ is the central density at the core radius $a_{0}$, and the exponent $\beta$ describes the density profile in the simplified King's (1972) model. Such a distribution of the ambient medium is assumed to be invariant with redshift.

Half of the cocoon (from the core to the hotspot) is approximated by a cylinder of length $L_{\text {jet }}=D_{\mathrm{s}} / 2$ and base diameter $b$. Here $D_{\mathrm{s}}$ is the total deprojected length (linear size) of the source. The ratio of the source's length to the base diameter is called the "axial ratio" $(A R)$; in the original KDA paper, half of the cocoon has an axial ratio of $R_{\mathrm{T}}=A R / 2$. The cocoon expands along the jet axis driven by the hotspot plasma pressure $p_{\mathrm{h}}$ and in the perpendicular direction by the cocoon pressure $p_{\mathrm{c}}$. The rate at which energy is transported from the AGN along each jet, i.e. the jet power $Q_{\text {jet }}$, is assumed to be constant during the source's lifetime. The model predicts self-similar expansion of the cocoon and gives analytical formula for the time evolution of various geometrical and physical parameters, e.g. the length of the jet (Eqs. (4) and (5) in KA)

$L_{\mathrm{jet}}(t)=c_{1}\left(\frac{Q_{\mathrm{jet}}}{\rho_{0} a_{0}^{\beta}}\right)^{1 /(5-\beta)} t^{3 /(5-\beta)}$,

and the cocoon pressure (Eq. (20) in KA)

$$
\begin{aligned}
p_{\mathrm{c}}= & \frac{18 c_{1}^{(2-\beta)}}{\left(\Gamma_{\mathrm{x}}+1\right)(5-\beta)^{2} \mathcal{P}_{\mathrm{hc}}}\left(\rho_{0} a_{0}^{\beta}\right)^{3 /(5-\beta)} \\
& \times Q_{\mathrm{jet}}^{(2-\beta) /(5-\beta)} t^{-(4+\beta) /(5-\beta)},
\end{aligned}
$$

where $c_{1}$ is a dimensionless constant (Eq. (25) in KA), $\Gamma_{\mathrm{x}}-$ the adiabatic index of the unshocked medium surrounding the cocoon, $t$ - the time elapsed since the jet started from the AGN, i.e. it is the source actual age and $\mathcal{P}_{\mathrm{hc}} \equiv p_{\mathrm{h}} / p_{\mathrm{c}}$ - the pressure ratio. In our calculations we use the empirical formula taken from K2000

$\mathcal{P}_{\mathrm{hc}}=(2.14-0.52 \beta) R_{\mathrm{T}}^{2.04-0.25 \beta}$. 


\subsection{Source energetics and radio power}

The energy delivered by the twin jets during the source lifetime is equal simply to $2 Q_{\text {jet }} t$. Accounting for the energy losses of relativistic particles due to adiabatic expansion within the timedependent volume of the cocoon $V_{\mathrm{c}}(t)$, the ratio of the jets' energy and the energy stored in the cocoon $E_{\text {tot }}$ is

$\frac{2 Q_{\mathrm{jet}} t}{E_{\mathrm{tot}}}=\frac{9 \Gamma_{\mathrm{c}}-4-\beta}{5-\beta}+\frac{9\left(\Gamma_{\mathrm{c}}-1\right)}{4(5-\beta)} \mathcal{P}_{\mathrm{hc}}$,

where $E_{\mathrm{tot}}(t)=u_{\mathrm{c}}(t) V_{\mathrm{c}}(t), u_{\mathrm{c}}=p_{\mathrm{c}}(t) /\left(\Gamma_{\mathrm{c}}-1\right)$ is the energy density in the cocoon, and $\Gamma_{\mathrm{c}}$ is the adiabatic index of the cocoon's matter.

Following KDA, we assume that the cocoon is composed of many small-volume elements filled with magnetized plasma and cosmic rays that are injected into the cocoon at the jet head the jet terminal shock. The initial energy distribution $n\left(\gamma_{i}\right)$ of injected particles with the Lorentz factor $\gamma_{\mathrm{i}}$ is assumed to have a single power-law form with the spectral index $p: n\left(\gamma_{\mathrm{i}}\right)=n_{0} \gamma_{\mathrm{i}}^{-p}$. The unknown normalization parameter $n_{0}$ is given by integrating the initial power-law distribution over the entire (assumed) energy range $\left[\gamma_{i, \min }, \gamma_{i, \max }\right]$.

The relativistic particles injected into the cocoon lose their energies in a magnetic field of energy density $u_{\mathrm{B}}$, and thus the cocoon's radio spectrum evolves with time. Tracing the effects of adiabatic expansion, synchrotron losses (with the assumed effective isotropization of the particles' pitch angle distribution), and inverse Compton scattering on the cosmic background radiation in the volume elements independently, the radio power of the cocoon $P_{v}$ at a fixed observing frequency $v$ is obtained by summing up the contributions from all volume elements, resulting in an integral over time (see Eq. (16) in KDA). It depends on the source's age $t$, redshift $z$, the jet power $Q_{\text {jet }}$, the half-cocoon axial ratio $R_{\mathrm{T}}$, the exponent in the initial power-law distribution of relativistic particles $p$, the values of the low-energy and highenergy cut-offs $\gamma_{i, \min }$ and $\gamma_{i, \max }$, and on the ratio $\zeta$ of the magnetic field energy $u_{\mathrm{B}}$ to the energy of relativistic electrons $u_{\mathrm{e}}$. The integral is not analytically solvable and has to be calculated numerically.

On the basis of the above analytical model, we aim to predict the dynamical age and the two physical parameters of the analyzed FR II-type radio sources: $Q_{\text {jet }}$ and $\rho_{0}$. In order to derive these parameters, all the other free parameters of the model $\left(\Gamma_{\mathrm{c}}\right.$, $\Gamma_{\mathrm{x}}, a_{0}, \beta, \zeta, p, \gamma_{\mathrm{i}, \min }, \gamma_{\mathrm{i}, \max }$ ) have to be fixed (see Table 1).

\subsection{Selection of the free model parameters}

Following the KDA analysis, we adopt their Case 3 where both the cocoon and the ambient medium are assumed to be described best by the "cold" equation of state $\left(\Gamma_{\mathrm{c}}=\Gamma_{\mathrm{x}}=5 / 3\right)$. For the initial ratio of the energy densities of the cocoon's magnetic field and particles, we use the equipartition condition $\zeta \equiv u_{\mathrm{B}} / u_{\mathrm{e}}=$ $(1+p) / 4$, which is well supported by the X-ray observations of the lobes in powerful radio sources (see Kataoka \& Stawarz 2005; Croston et al. 2005, and references therein).

Selection of the particular (universal) values regarding external medium parameters $a_{0}$ and $\beta$ requires some justification. We note that even careful 2-D modeling of a distribution of radio emission for powerful sources with quite regular structure can lead to values of $a_{0}$ that are discrepant with those implied by the X-ray observations of the intergalactic thermal environment. In our simplified approach, we assume $a_{0}=10 \mathrm{kpc}$ for all the analyzed sources, a conservative value between $2 \mathrm{kpc}$ used by KDA, and the $50 \mathrm{kpc}$ found by Wellman et al. (1997). We
Table 1. Observational and model parameters (see Sects. 2.1-2.3 for detailed descriptions).

\begin{tabular}{|c|c|c|}
\hline & Symbol & Dimension \\
\hline Observational & $z$ & [dimensionless] \\
\hline parameters & $D$ & {$[\mathrm{kpc}]$} \\
\hline derived & $A R$ & [dimensionless] \\
\hline from radio maps & $v$ & {$[\mathrm{MHz}]$} \\
\hline and spectrum & $P_{v}$ & {$\left[\mathrm{~W} \mathrm{~Hz}{ }^{-1} \mathrm{sr}^{-1}\right]$} \\
\hline Model free & $a_{0}$ & {$[\mathrm{kpc}]$} \\
\hline parameters & $\beta$ & [dimensionless] \\
\hline \multirow[t]{6}{*}{ (to be fixed) } & $p=2 \alpha_{\mathrm{inj}}+1$ & [dimensionless] \\
\hline & $\gamma_{i, \min }, \gamma_{i, \max }$ & [dimensionless] \\
\hline & $\Gamma_{\mathrm{c}}, \Gamma_{\mathrm{x}}$ & [dimensionless] \\
\hline & $\zeta$ & [dimensionless] \\
\hline & $t$ & {$[\mathrm{yr}]$} \\
\hline & $\theta$ & [deg] \\
\hline Model parameters & $Q_{\text {jet }}$ & {$[\mathrm{W}]$} \\
\hline derived for given & $\rho_{0}$ & {$\left[\mathrm{~kg} \mathrm{~m}^{-3}\right]$} \\
\hline values of $t$ & $p_{\mathrm{c}}$ & {$\left[\mathrm{N} \mathrm{m}^{-2}\right]$} \\
\hline \multirow[t]{2}{*}{$\alpha_{\mathrm{inj}}$ and $\gamma_{\mathrm{i}, \max }$} & $u_{\mathrm{c}}$ & {$\left[\mathrm{J} \mathrm{m}^{-3}\right]$} \\
\hline & $E_{\text {tot }}$ & {$[\mathrm{J}]$} \\
\hline
\end{tabular}

also use a constant value of $\beta=1.5$, in agreement with Daly (1995), Blundell et al. (1999), Willott et al. (1999), and K2000. Note that the assumed ambient medium profile is then slightly flatter than the one adopted in the original KDA paper on the basis of the Canizares et al. (1987) analysis, which found $\beta=1.9$ to be typical at about $100 \mathrm{kpc}$ from the galactic nucleus (but see Gopal-Krishna \& Wiita 1987).

Another free parameter of the model is the orientation of the jet axis to the observer's line of sight $\theta$. We assume $\theta=90^{\circ}$ for the giant-size radio galaxies and $\theta=70^{\circ}$ for 'normal'-size radio galaxies. This latter value is justified on the basis of the unified scheme for extragalactic radio sources. In this scheme, the average orientation angle for radio galaxies is $\left\langle\theta_{\mathrm{RG}}\right\rangle \simeq 69^{\circ}$ (Barthel 1989). The apparent size $D$ of a radio source then yields the model cocoon length

$L_{\text {jet }}=D /(2 \sin \theta)$.

The next parameters that significantly influence the model predictions (as will be shown and discussed in the next sections) are the initial power-law exponent $p$, as well as the low- and highenergy cut-offs of the initial electron energy distribution, $\gamma_{i, m i n}$ and $\gamma_{\mathrm{i}, \max }$.

The cut-off energies in the electron energy distribution injected by the jet terminal shock to the expanding cocoon are poorly constrained by both observations and theory. The values considered in the literature for $\gamma_{\mathrm{i} \text {,max }}$ vary from $10^{4}$ up to $\geq 3 \times 10^{8}$ (see Barai \& Wiita 2006). Here we initially studied a very broad range $\gamma_{i, \max }=10^{4}-10^{10}$ (Sect. 4), in order to check how the results of the proposed method depend on the choice of this particular model parameter. We found that the effect is minor and note in this context that the high values of the maximum Lorentz factors for electrons accelerated at the jet terminal shocks are more likely, since a number of hotspots in powerful radio sources are established sources of synchrotron X-ray emission, meaning that production of high-energy $\left(\gamma_{i} \geq 10^{7}\right)$ electrons thus seems to be a general property of radio galaxies and quasars (see discussion in Hardcastle et al. 2004; Kataoka $\&$ Stawarz 2005, and references therein). For these reasons, later on in the analysis (Sect. 5) we consider a slightly narrower range of $\gamma_{i, \max }=10^{7}-10^{8.5}$.

As for the minimum electron energy, we note that most authors assume $\gamma_{\mathrm{i} \text {,min }} \rightarrow 1$, although such a choice is made largely 
for the sake of simplicity. Interestingly, Blundell et al. (2006) recently argued that the X-ray observations of giant radio galaxy 4C 39.24 indicate an initial electron low-energy cut-off as high as $\gamma_{i, \text { min }} \sim 10^{4}$; see also in this context Carilli et al. (1991) for the low-frequency radio observations of Cyg A hotspots. We believe, however, that these data do not unambiguously show any sharp low-energy cut-off of the electron energy distribution at some particular (ultrarelativistic) energy, but instead indicate only spectral flattening at low $\left(\gamma_{\mathrm{i}, \mathrm{min}}<10^{4}\right)$ electron energies. Thus, hereafter we take $\gamma_{\mathrm{i}, \mathrm{min}}=1$, assuming that the particles at the jet terminal shock are picked up by the acceleration process(es) directly from the "thermal pool" of the cold plasma carrying the bulk of the jet kinetic energy to form the non-thermal electron energy distribution injected further into the expanding cocoon. We checked, however, that taking $\gamma_{i, \min }=10$ (as favored by the analysis of Barai \& Wiita 2006) does not change our results obtained with $\gamma_{\mathrm{i}, \min }=1$ as presented in the next sections.

These choices regarding low- and high-energy electron cutoffs seem to be justified, although they may lead to inconsistency with the main assumption of the model, namely with the assumed single power-law form of the initial electron energy distribution. If the low-energy spectral flattening of the electron spectrum is the case (as has to be accepted if indeed $\gamma_{i, \min }=1$ ), it suggests that the particle distribution injected by the jet terminal shocks to the lobes/cocoons is characterized by some more complex spectral shape, for which a broken power-law could be for example a simple approximation. Meanwhile, in the original KDA model, universal single power-law initial energy distribution of the relativistic particles with the energy index $p=2.14$ was assumed, corresponding to the radio spectral index $\alpha_{\text {inj }}=0.57$. This value agrees perfectly with the one obtained for the classical FR II-type radio source Cyg A by means of fitting its radio spectrum by the "continuum injection" (C.I.) model (for the spectral aging theory review see Myers \& Spangler 1985; Carilli et al. 1991). It should be noticed that $p=2.14$ is close to, although not exactly equal to, the model spectral index of relativistic particles accelerated at non-relativistic shocks by the 1st-order Fermi (diffusive) acceleration process.

Most importantly, the low-frequency radio observations of a number of powerful radio sources imply a wide spread of the injection spectral index, $0.38 \leq \alpha_{\text {inj }} \leq 0.95$. This indicates that there may be no universal value to this parameter for all cocoons. Moreover, we note that the low-frequency radio spectra usually steepen with redshift (see Laing \& Peacock 1980; Macklin 1982; Gopal-Krishna 1988; van Breugel \& McCarthy 1990). The physical reason for such a dependence is unclear, although the effect seems to be real. Therefore, keeping in mind all the uncertainties regarding $\alpha_{\text {inj }}$ (or equivalently $p$ ), which reflect our poor understanding of the particle acceleration processes taking place at mildly relativistic collisionless astrophysical shocks, and also the observational limitations, we decided to treat the injection spectral index as a free parameter, which may be different for different sources.

We also emphasize that the particular value of power-law index $p$ obtained for a given source with the fitting procedure does not necessarily correspond to some well-defined acceleration process taking place at the jet terminal shock. Instead, it should be considered as an effective spectral index of the injected electron continuum (characterized most likely by more complex spectral shape), averaged over a very broad energy range and over the lifetime of the source. This issue will be discussed further in Sect. 6.

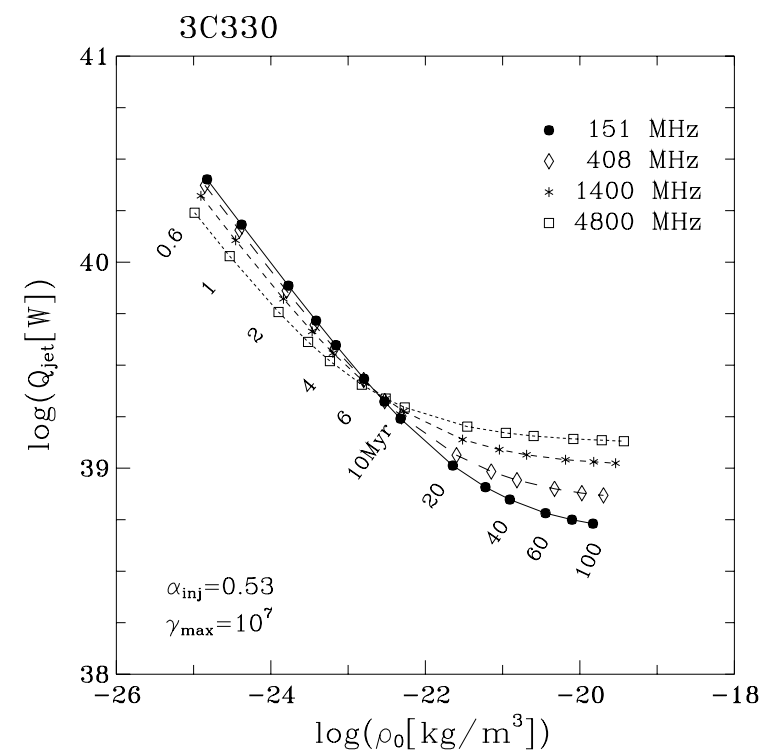

Fig. 1. A set of $Q_{\text {jet }}(t)$ and $\rho_{0}(t)$ solutions at the four observing frequencies, for a number of the age values assumed for the radio galaxy 3C 330 .

\subsection{The fitting procedure}

In Table 1 we summarize all the observational and model parameters characterizing the source's cocoon that are used in the present calculations.

Assuming a value for the source age $t$ and having fixed all the free parameters of the model as discussed in Sect. 2.3, we find the jet power $Q_{\text {jet }}$ and the central density of the external medium $\rho_{0}$ for an investigated source by an iterative solution of the system of two equations: (i) Eq. (1) equated to Eq. (3) for the jet length $L_{\text {jet }}$ and (ii) the integral for the luminosity of the cocoon $P_{v}$ (Eq. (16) in KDA, see Sect. 2.2). These equations require matches of the solution to the observed values of $D$ to (i) and $P_{v}$ to (ii) at a number of different frequencies, respectively. This fitting procedure proved to be stable and always provides a unique solution.

Performing the above calculations ${ }^{1}$ for a number of age values $t$ assumed for a given source, we obtain a set of solutions for $Q_{\text {jet }}(t)$ and $\rho_{0}(t)$, which show a correlation of $Q_{\text {jet }}$ and anticorrelation of $\rho_{0}$, as expected, with the source age $t$ for a given apparent size $D$ and total cocoon luminosity $P_{v}$. An example of this effect for the four observing frequencies is shown in Fig. 1. A perfect intersection of all the $Q_{\text {jet }}-\rho_{0}$ curves corresponding to different observing frequencies at some particular "true" age of a source is expected if the observed shape of its total radio spectrum (used here to determine the radio power at different frequencies) agrees with the theoretically predicted one in the framework of the C.I. model and if the dynamical (KDA) model applied here is indeed correct. However, the observed spectrum of a number of the analyzed sources evidently departs from the predicted behavior. In such cases, the sources' high-frequency spectra are steeper than expected, so that the resultant $Q_{\text {jet }}-\rho_{0}$ curve corresponding to the highest observing frequency bisects other curves at the age inconsistent with what is implied by the remaining ones.

\footnotetext{
1 Our program DYNAGE for calculating the model parameters $\left(Q_{\text {jet }}\right.$, $\rho_{0}, p_{\mathrm{c}}, u_{\mathrm{c}}$, and $\left.E_{\mathrm{tot}}\right)$ for a set of given values of $t, \alpha_{\mathrm{inj}}$, and $\gamma_{\mathrm{i} \text {,max }}$, as described in this paper and illustrated in Fig. 1, is available upon request from J. Machalski (machalsk@oa.uj .edu.pl).
} 
A "goodness" of the intersection is quantified by the $\Delta$ measure, which is defined as follows: indicating $\log \rho_{0}(t) \equiv x(t)$ and $\log Q_{\text {jet }}(t) \equiv y(t)$, we calculate the mean separation between the points determined by the values of $[x(t), y(t)]$ in the $\log Q_{\text {jet }}-$ $\log \rho_{0}$ plane as

$\Delta(t)=\frac{2}{n(n-1)}$

$\times \sum_{i=1}^{n-1}\left(\sum_{j=i}^{n-1}\left\{\left[x_{i}(t)-x_{j+1}(t)\right]^{2}+\left[y_{i}(t)-y_{j+1}(t)\right]^{2}\right\}^{1 / 2}\right)$,

where $n$ is the number of the observing frequencies included. A minimum of $\Delta(t)$ (i.e. "the best" intersection of the $Q_{\text {jet }}-\rho_{0}$ curves for different frequencies) is considered as an indicator of the source's real dynamical age $t$. This minimum also distinguishes a unique values of $Q_{\text {jet }}$ and $\rho_{0}$, as well as $p_{\mathrm{c}}$ and $E_{\text {tot }}$, for the considered source. Hereafter, the age corresponding to this set of $Q_{\text {jet }}$ and $\rho_{0}$ values is called the age solution.

However, the calculations indicate that the "age solution" depends significantly on the exponent in the initial power-law energy distribution, $p$. In addition, they suggest that there is no canonical value of this injection spectral index that would be good for each and every source. (This in fact agrees with the preliminary notes given at the end of the previous section; this issue will be discussed further in Sect. 6.) For this reason, the best value of $p$ (or equivalently $\alpha_{\text {inj }}$ ), needed for a precise estimate of the dynamical age of a particular FR II-type radio source, is determined below by finding the minimum of the jet kinetic energy delivered to the cocoon (see Sect. 4.3). The age solution corresponding to the minimum jet energy is hereafter called the best solution.

\section{The data}

The data necessary for determining the age of a given radio source with the method presented above are: its projected linear length $D$, its axial ratio $A R$, its total radio power $P_{v}$ at a number of observing frequencies, and the source's redshift $z$.

As we limit our method to FR II-type sources, $D$ is invariant of frequency and can be precisely determined from the radio maps. The lateral size (base diameter $b$ of the cocoon) is taken as the average of the widths of the two lobes. They are determined on the maps with the highest sensitivity to surface brightness as the deconvolved width of a transversal cross-section through the lobes measured halfway between the core and the outer edge of the source. Thus, $A R=D / b$.

The source power $P_{v}$ is determined at four frequencies: $v=151,408,1400$, and $4800 \mathrm{MHz}$. Because not all the sources were observed at these particular frequencies, the cocoon's spectrum was derived from the available flux densities with the contributions from the radio core and the hotspots subtracted whenever possible. Next, the simple analytical functions $y=$ $a+b x+c \exp ( \pm x)$, where $x=\log v[\mathrm{GHz}]$ and $y=\log S(v)[\mathrm{mJy}]$, were fitted to these flux densities weighted by their given error. These two functions were found to provide the best fit (the least $\left.\chi^{2}\right)$ to the data. Then, the values of $P_{151}, P_{408}, P_{1400}$, and $P_{4800}$ were calculated using flux densities found with the above functions and the appropriate radio K-corrections ${ }^{2}$.

${ }^{2}$ There is no need to use the same frequencies for different sources; a few observing frequencies should cover a wide range of the radio spectrum.

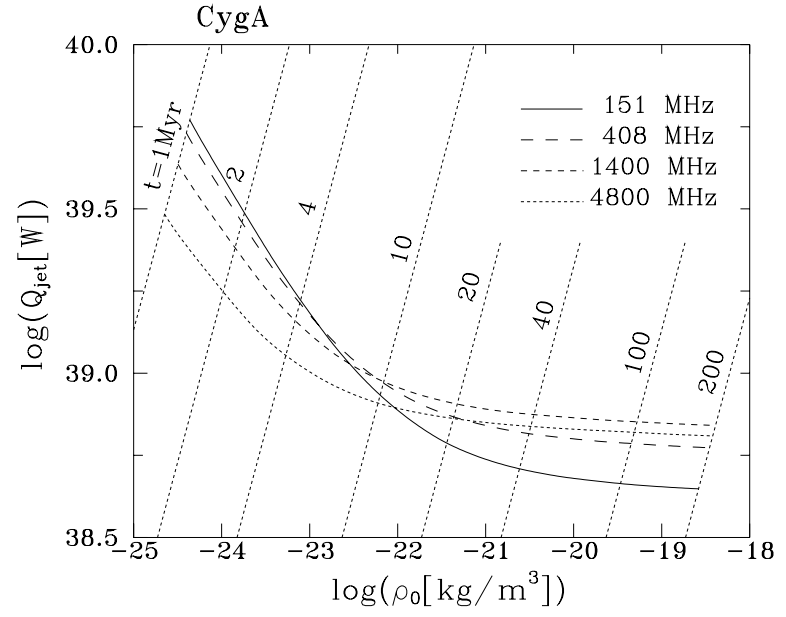

Fig. 2. $Q_{\text {jet }}-\rho_{0}$ diagram for Cyg A at the four observing frequencies. The dotted diagonal lines indicate an age related to the fitted $Q_{\text {jet }}$ and $\rho_{0}$ values. Other free parameters for all the curves are: $R_{\mathrm{T}}=1.95, \beta=1.5$, $\alpha_{\text {inj }}=0.57$, and $\gamma_{\mathrm{i}, \max }=10^{4}$ (see also Sect. 2.3)

The FR II-type radio sources analyzed in this paper and their observational parameters as used in the fitting procedure are given in Table 2. These sources, subjectively selected from the sample of Machalski et al. (2004a), include high-redshift and low-redshift, high-luminosity and low-luminosity, giant-sized and normal-sized radio galaxies. The sources in Table 2 do not include quasars whose jets' axes (and hence radio structures) may be strongly projected onto the sky.

\section{Fitting result for Cyg A}

In this section the dynamical age estimate and its dependence on the values of the model free parameters are presented for Cyg A.

\subsection{The age solution and its dependence on $\alpha_{\text {inj }}$ and $\gamma_{\mathrm{i}, \max }$}

Assuming $\theta=80^{\circ}$ and adopting (after K2000) parameters $p=2.14$ and $\gamma_{\mathrm{i}, \max }=10^{4}$, the fitted values of $Q_{\mathrm{jet}}$ and $\rho_{0}$ for different hypothetical ages of this source are shown as the $\log Q_{\text {jet }}-$ $\log \rho_{0}$ diagram in Fig. 2. This figure shows that the four curves corresponding to the four observing frequencies intersect at the age of about $10 \mathrm{Myr}$. The age solution giving a minimum of the $\Delta(t)$ measure for the set of the model free parameters (recalled in the caption of Fig. 2) is $t=10.9 \pm 0.9 \mathrm{Myr}$.

As mentioned previously, a minimum of $\Delta(t)$ appears to be dependent on the assumed parameters of the initial energy distribution of relativistic particles. The dependence of $\Delta$ on $t$ for two different (rather extreme) values of $\gamma_{i, \max }=10^{4}$ and $=10^{10}$ is plotted in Fig. 3 for different values of $\alpha_{\text {inj }}$. As shown, the age solution is not very sensitive to the particular choice of $\gamma_{i, \max }$, especially if $\alpha_{\text {inj }}>0.5$.

The dependence of the resulting age solution on $\alpha_{\text {inj }}$ for the same two extreme values of $\gamma_{i, \max }$ is shown in Fig. 4. It is clear that the age solution increases strongly with the decreasing $\alpha_{\mathrm{inj}}$, and hence that the minimum $\Delta$ measure is smaller for higher

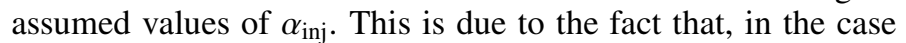
of flatter and flatter initial electron energy distributions, more and more time has to elapse for the radio continuum to steepen up to some given (observed) level. As a result, the obtained age 
Table 2. List of sources and their observational parameters derived from radio maps and spectra with the radio power in units of $\mathrm{W} \mathrm{Hz} \mathrm{Hr}^{-1}$.

\begin{tabular}{llrrrcccc}
\hline \hline Source name & $z$ & $D[\mathrm{kpc}]$ & $A R$ & $\theta\left[^{\circ}\right]$ & $\log P_{151}$ & $\log P_{408}$ & $\log P_{1400}$ & $\log P_{4800}$ \\
\hline Cyg A & 0.0564 & 135 & 3.9 & 80 & 27.796 & 27.465 & 26.963 & 26.358 \\
3C55 & 0.7348 & 496 & 6.4 & 70 & 27.644 & 27.247 & 26.718 & 26.148 \\
3C103 & 0.330 & 458 & 7.0 & 70 & 26.906 & 26.630 & 26.140 & 25.552 \\
3C165 & 0.296 & 384 & 3.4 & 70 & 26.579 & 26.244 & 25.769 & 25.227 \\
3C239 & 1.786 & 96 & 2.7 & 70 & 28.483 & 28.092 & 27.541 & 26.914 \\
3C247 & 0.749 & 95 & 4.8 & 70 & 27.350 & 27.117 & 26.746 & 26.284 \\
3C280 & 0.996 & 113 & 2.9 & 70 & 27.990 & 27.697 & 27.288 & 26.829 \\
3C292 & 0.71 & 960 & 8.4 & 90 & 27.406 & 27.020 & 26.547 & 26.075 \\
3C294 & 1.779 & 135 & 3.8 & 70 & 28.412 & 27.984 & 27.431 & 26.841 \\
3C322 & 1.681 & 283 & 5.0 & 70 & 28.171 & 27.906 & 27.485 & 26.954 \\
3C330 & 0.549 & 395 & 10.9 & 90 & 27.432 & 27.150 & 26.771 & 26.334 \\
3C332 & 0.1515 & 229 & 4.9 & 70 & 25.744 & 25.460 & 25.068 & 24.631 \\
B0908+376 & 0.1047 & 72 & 2.3 & 70 & 24.694 & 24.452 & 24.120 & 23.755 \\
B1209+745 & 0.107 & 795 & 5.8 & 90 & 24.919 & 24.583 & 24.152 & 23.706 \\
B1312+698 & 0.106 & 794 & 5.5 & 90 & 25.174 & 24.883 & 24.497 & 24.066 \\
\hline
\end{tabular}

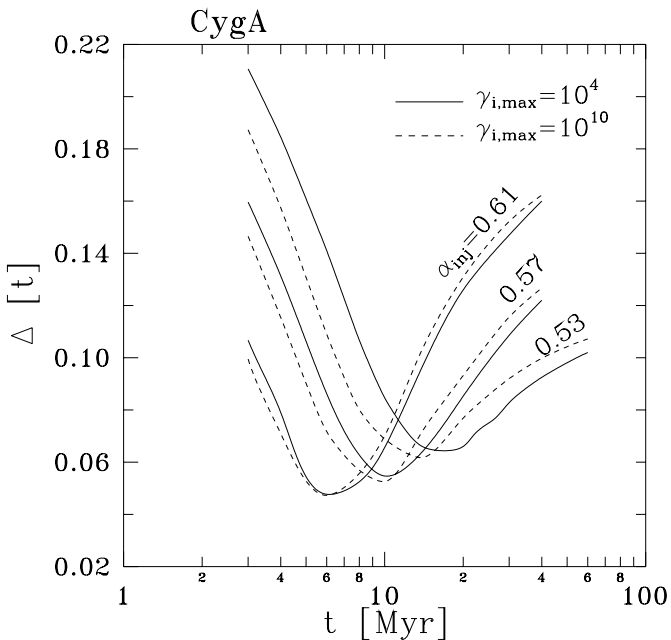

Fig. 3. The influence of the initial energy distribution parameters on the "goodness" of fit quantified by the $\Delta$ measure for Cyg A.

solution anticorrelates with the injection spectral index, meaning that the minimum of $\Delta$ also decreases with decreasing $t$. Such a decrease continues until reaching the point where the radio continuum does not have time to steepen at all, corresponding to the situation where all the curves on the $Q_{\text {jet }}-\rho_{0}$ overlap each other. Such an ambiguous situation leads to the question of which value of $\alpha_{\text {inj }}$ one should really assume for a given source to apply our method of the dynamical age estimate. This issue will be addressed further in Sect. 4.3 below.

\subsection{Dependence on $\beta, A R$, and $\theta$}

The fitting results indicate that the effect due to changing particular values of the $\beta, A R$, and $\theta$ parameters is of minor importance for the resulting age solution. Figure 5 shows the influence of the exponent $\beta$ in the density distribution of the ambient medium and of the source axial ratio $A R \equiv R_{\mathrm{T}} \times 2$ on the age estimate. (Though the $A R$ parameter is the observed one, it is much less precisely measured than the source linear size $D$.). The influence of the unknown inclination angle $\theta$ on the age is also small. Comparison of Eqs. (1) and (3) yields $t \propto(D / \sin \theta)^{(5-\beta) / 3}$. Thus, $t$ increases no more than 1.21 and 1.15 for $\beta=1$ and $\beta=2$, respectively, if $\theta$ decreases from $90^{\circ}$ to $60^{\circ}$. We conclude that

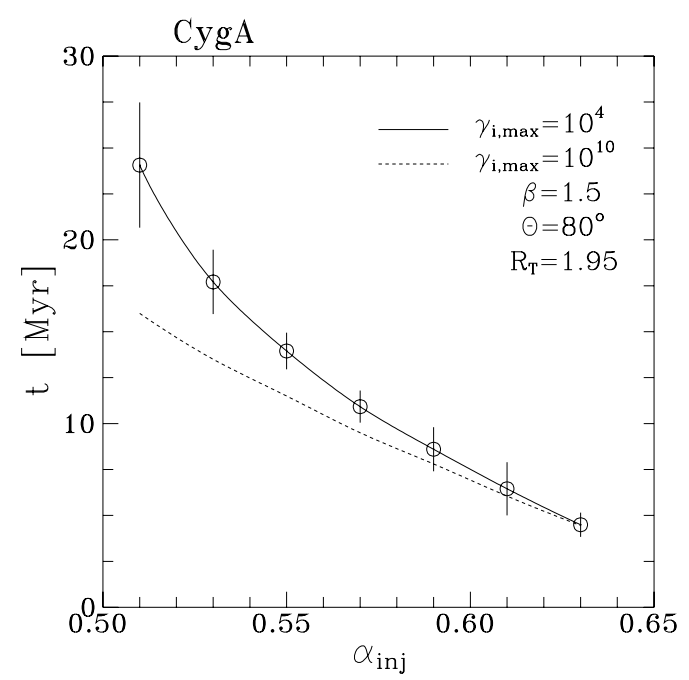

Fig. 4. The influence of $\alpha_{\text {inj }}$ on the age estimate for Cyg A, with a set of the fixed model parameters.

the influence of $\theta$ on $t$ is comparable to that caused by the uncertainty in the $A R$ determination.

\subsection{Dependence of the age solution on the kinetic energy delivered by the jet}

As noted in Sect. 4.1, the fitted jet power increases with decreasing age (hence with increasing $\alpha_{\text {inj }}$ ) for a given size and brightness of the source (see Figs. 1 and 2). In Fig. 6 we show both the fitted jet power and the age of Cyg A as a function of $\alpha_{\text {inj }}$ for the two extreme values of $\gamma_{i, \max }$ considered in this section. As the parameters $Q_{\text {jet }}$ and $t$ depend on $\alpha_{\text {inj }}$ in the opposite way, there is always a minimum value of the product $Q_{\text {jet }} \times t$. This product gives the kinetic energy delivered to the cocoon by the jet. The fitting procedure thus indicates that this total energy has a minimum for some specific value of $\alpha_{\text {inj }}$ and fixed observational parameters.

Based on the above effect, we consider the age corresponding to the minimum kinetic energy as the "best solution" for the dynamical age of a given source (see Sect. 2.4). The "best solution" for Cyg A gives $t=10.4 \pm 1.6 \mathrm{Myr}\left(\alpha_{\mathrm{inj}}=0.574 \pm 0.011\right)$ and $t=5.7 \pm 0.4 \mathrm{Myr}\left(\alpha_{\mathrm{inj}}=0.621 \pm 0.007\right)$ for $\gamma_{\mathrm{i}, \max }=10^{4}$ 


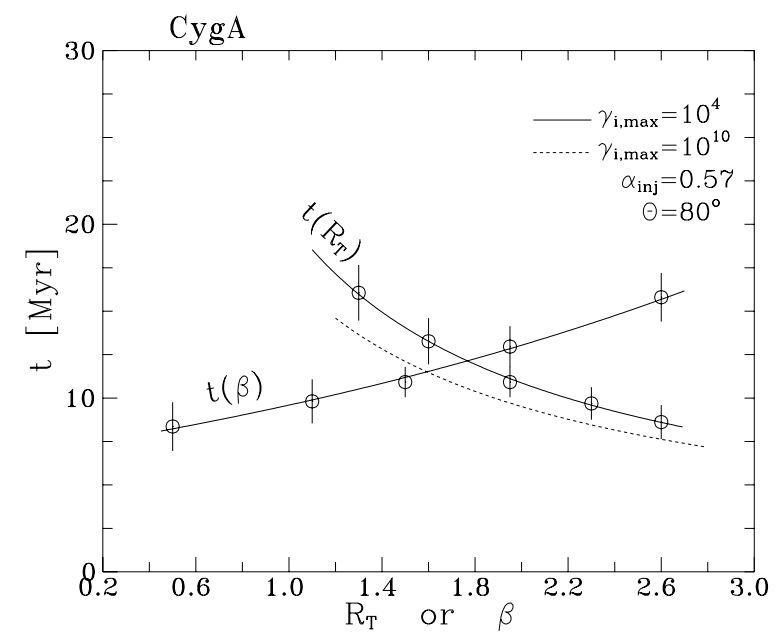

Fig. 5. The influence of $\beta$ and $R_{\mathrm{T}}$ on the age estimate for Cyg A, with a set of the fixed model parameters.

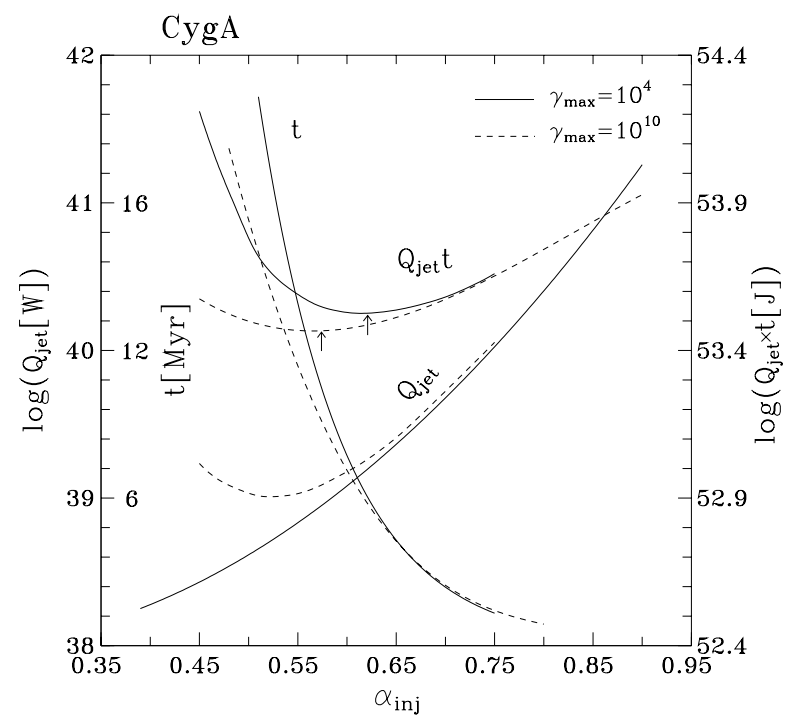

Fig. 6. Diagram of the jet power $Q_{\text {jet }}$, the age $t$, and the total energy $Q_{\text {jet }} t$ delivered by the Cyg A jet to its cocoon, as functions of $\alpha_{\text {inj }}$ for the two (extreme) values of $\gamma_{\mathrm{i}, \max }$. The arrows indicate $\alpha_{\text {inj }}$ values corresponding to the minimum total cocoon energy.

and $\gamma_{i, \max }=10^{10}$, respectively. Since one should expect in reality $\gamma_{i, \max }$ in between of the two extreme values considered here for illustrative purposes, we safely conclude that this method indicates that the age and the injection spectral index of Cyg A is $t=6-10 \mathrm{Myr}$ and $\alpha_{\mathrm{inj}}=0.57-0.62$, respectively, in very good agreement with other dynamical and spectral estimates published in the literature (see, e.g., Kino \& Kawakatu 2005). In addition, the corresponding kinetic power of the Cyg A jet is $Q_{\text {jet }}=(1-2) \times 10^{39} \mathrm{~W}$, which is a factor of 2-3 higher than the value estimated by $\mathrm{K} 2000$ and a factor of 2 lower than the one obtained by Kino \& Takahara (2004) by means of detailed analysis of the Cyg A hotspots' radiative properties.

\section{Fitting results for other radio galaxies}

Further results of the age estimation derived with our method are presented in more detail for two other sources: giant radio galaxy B 1312+698 and normal-sized galaxy 3C 55 .

\subsection{The "best solution" for the age}

The appropriate $Q_{\text {jet }}$ vs. $\rho_{0}$ diagrams for the given values of $\alpha_{\text {inj }}$ and $\gamma_{i, \max }$ are shown in Figs. $7 \mathrm{a}$ and $8 \mathrm{a}$, with an age related to the values of $Q_{\text {jet }}$ and $\rho_{0}$ fitted to observations at the four different frequencies. In this case, the ideal intersections of the four curves give the precise "age solutions", as well as the precise estimates of the jet powers and the central core densities for both sources.

The dependence of these "age solutions" (the curves marked by $t$ ) on $\alpha_{\text {inj }}$ and $\gamma_{\mathrm{i} \text {,max }}$ is presented in Figs. $7 \mathrm{~b}$ and $8 \mathrm{~b}$, together with the $Q_{\text {jet }}-\alpha_{\text {inj }}$ and $Q_{\text {jet }} t-\alpha_{\text {inj }}$ curves. Clearly, the "best solutions" exist within the theoretically acceptable range of $0.5 \leq \alpha_{\text {inj }} \leq 0.7$.

The "best solutions" for the dynamical age of all the sources from Table 2 with the corresponding values of $\alpha_{\text {inj }}$ are collected in Table 3. In order to show how these solutions depend on the assumed value of the maximum electron energies, we give the best solution for $\gamma_{i, \max }=10^{7}$ and $3 \times 10^{8}$. As shown, the differences are minor. In particular, Col. 6 of Table 3 gives the ratio of the standard deviation to the mean of the ages derived with these two different values $\gamma_{i \text {, max }}$, showing that the resulting age estimates differ from $\sim 4 \%$ to $\sim 30 \%$, mostly by only $\sim 10 \%$.

\subsection{Comparison of the "best solution" dynamical ages with the synchrotron ages}

One quality of the dynamical age estimated with the method presented here can be justified by its comparison with the synchrotron (radiative) age, $\tau_{\text {rad }}$, derived from the spectral aging analysis (e.g. Myers \& Spangler 1985; Carilli et al. 1991). In this analysis the radiative age of emitting particles is determined from the "break" frequency in the observed radio spectrum and the magnetic field strength, usually computed under the equipartition conditions. In order to find this break frequency, the $\alpha_{\text {inj }}$ value must be known. In most of the published papers concerning the above analysis, the value of $\alpha_{\text {inj }}$ was usually identified with the slope of the observed low-frequency spectrum. Because of a reasonable criticism of this simple method (see Sect. 1), Murgia (1996) developed the software SYNAGE, which allows the best fit of the spectral data to the theoretical models of the energy losses by constraining the values of the most important model parameters, especially a value of $\alpha_{\text {inj }}$.

The $\alpha_{\text {inj }}$ value for the C.I. model, $\alpha_{\mathrm{CI}}$, fitted to the spectrum of the lobes of the sources from Table 2 using the SYNAGE algorithm, is given in Col. 7 of Table 3 . It is easy to see that the values of $\alpha_{\mathrm{CI}}$ are typically higher than the values of $\alpha_{\text {inj }}$ determined by minimizing the total energy as proposed in this paper, though the errors in $\alpha_{\text {inj }}$ are of the same order as the errors in $\alpha_{\mathrm{CI}}$ (also given in Col. 7). Only for Cyg A and (formally) for 3C 165 are the fitted values of $\alpha_{\mathrm{CI}}$ lower than the values of $\alpha_{\mathrm{inj}}$.

A discrepancy between the values of $\alpha_{\mathrm{CI}}$ and $\alpha_{\text {inj }}$ for the other sources can be explained either by (i) a lack of flux density data at frequencies below $\sim 100 \mathrm{MHz}$ (which may be crucial for a proper determination of the slope of the initial spectrum) and the uncertainties of the available data or by (ii) an influence of other physical processes not taken into account in the KDA model, such as reacceleration of the relativistic particles, nonstandard evolution of the cocoon's magnetic fields, etc. Note that the $\alpha_{\mathrm{CI}}$ values, fitted with the SYNAGE and much higher than the effective values of $\alpha_{\text {inj }}$ determined by the minimum jet energy delivered to the source (cocoon), may lead to a most unlikely young age and a corresponding enormously high jet-head advance velocity, $v_{\mathrm{h}}$. For example, $\alpha_{\mathrm{inj}} \approx \alpha_{\mathrm{CI}}=0.898$ for the highredshift radio galaxy 3C 294 (see Table 3) would only yield the 

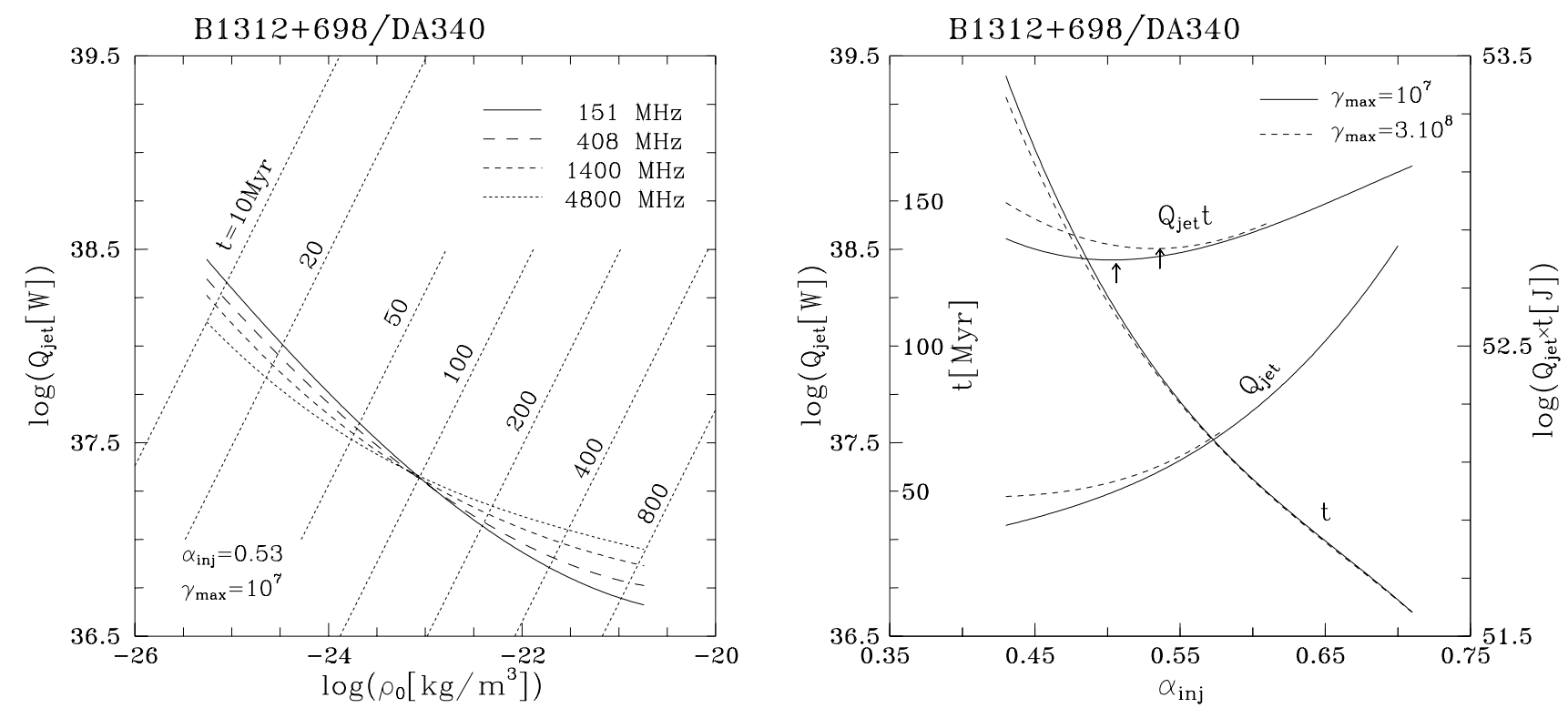

Fig. 7. a) $Q_{\text {jet }}-\rho_{0}$ diagram for the giant radio galaxy B $1312+698$ : the "age solution" for $\alpha_{\text {inj }}=0.53$ and $\gamma_{\text {i,max }}=10^{7}$. b) $Q_{\text {jet }}, t$, and $Q_{\text {jet }} \times t$ vs. $\alpha_{\text {inj }}$ diagrams for two different values of $\gamma_{\mathrm{i}, \max }$. The minima of the total energy corresponding to the "best solution" are marked with arrows.
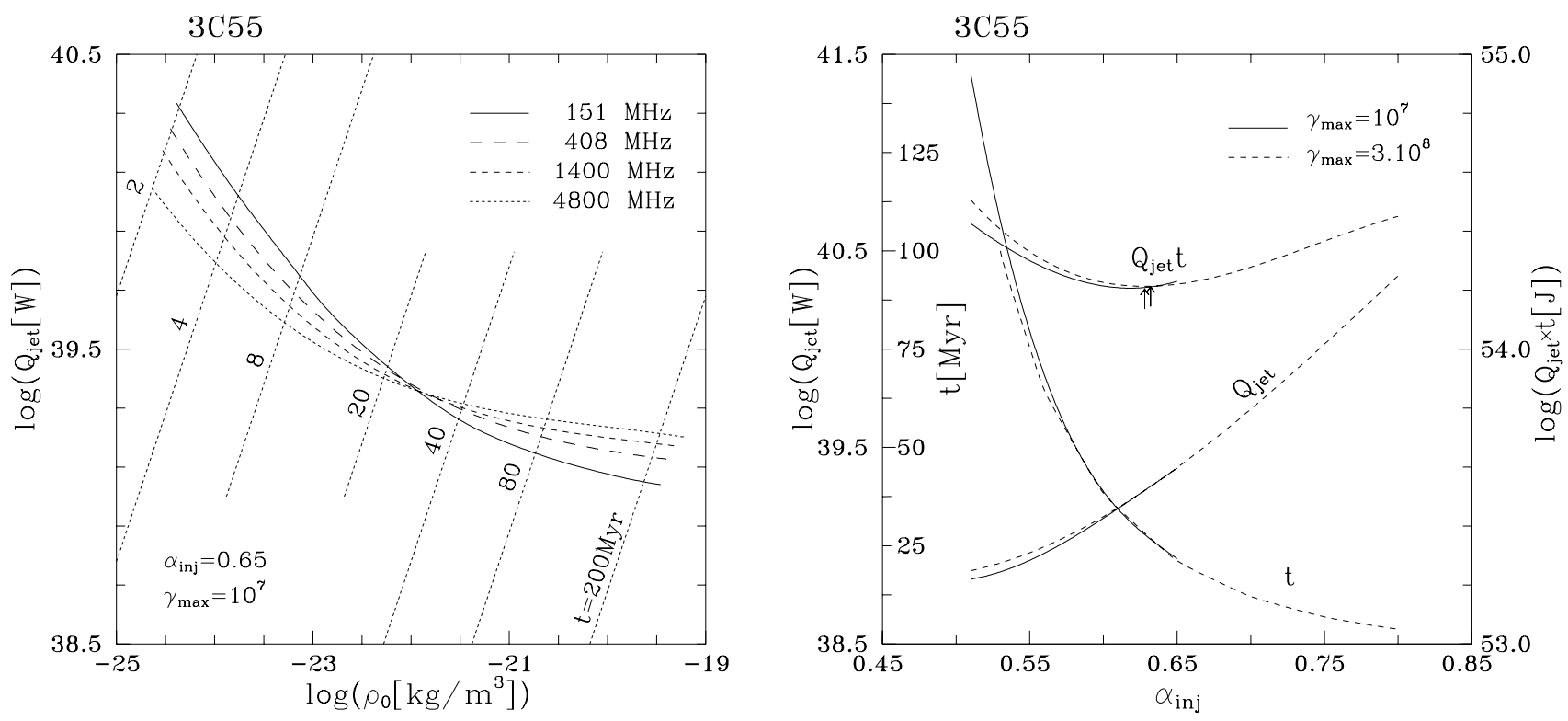

Fig. 8. The same as in Fig. 7 but for the radio galaxy 3C 55.

dynamical age of $0.25 \mathrm{Myr}$, which would imply the unacceptable high value of $v_{\mathrm{h}}=0.936 c$ !

We note in this context that high $(\sim 0.3 \mathrm{c})$ advance velocities are, in fact, observed, however only in "compact symmetric objects" (CSOs; see, e.g., Owsianik \& Conway 1998; Polatidis \& Conway 2003; Gugliucci et al. 2005). These sources, characterized by projected linear sizes less than $\sim 1 \mathrm{kpc}$, are believed to be young versions of the extended radio galaxies, possibly even of the powerful classical doubles (see a review by O'Dea 1998). And indeed, the implied kinetic ages (between $100 \mathrm{yr}$ and $10^{4} \mathrm{yr}$ ) usually agree closely with the spectral ages determined for some of these objects assuming energy equipartition within the lobes (Katz-Stone \& Rudnick 1997). On the other hand, it should not be surprising that the advanced velocities of the jets in CSOs are higher than the ones in "regular" FR II radio

galaxies, for which Scheuer (1995) gives an upper limit of $0.1 c$. That is because of the differences in the lobe/jet evolution for compact and extended objects, caused for example by the differences in the ambient medium density profiles. In particular, Kawakatu \& Kino (2006) argue for constant jet-advanced velocity of evolving young/compact radio sources, while for the decreasing advanced velocity with increasing linear size at later evolutionary stages (see also in this context Alexander 2000). Therefore, here we take $0.3 c$ as an upper limit for the "realistic" values of the jet-advanced velocities in all the FR II radio galaxies considered in this paper, in agreement with Scheuer (1995), and multi-epoch observations of young radio sources.

Finally, the synchrotron ages $\tau_{\text {rad }}$ (with associated errors) for all the sources in Table 2 - collected from the papers of 
Table 3. $\alpha_{\text {inj }}$ values relating to the minimum total energy of the jet for two different values of $\gamma_{\mathrm{i}, \mathrm{max}}$, and the corresponding "best solution" of age for the analyzed sample of FR II-type radio galaxies.

\begin{tabular}{lcccccccc}
\hline \hline & $\gamma_{\max }=10^{7}$ & \multicolumn{3}{c}{$\gamma_{\max }=3 \times 10^{8}$} & & & \\
Source & $\alpha_{\text {inj }}$ & $t[\mathrm{Myr}]$ & $\alpha_{\text {inj }}$ & $t[\mathrm{Myr}]$ & $\Delta t / \bar{t}$ & $\alpha_{\mathrm{CI}}$ & $\tau_{\text {rad }}[\mathrm{Myr}]$ & $t / \tau_{\text {rad }}$ \\
1 & 2 & 3 & 4 & 5 & $6^{*}$ & $7 * *$ & $8^{* * *}$ & 9 \\
\hline Cyg A & 0.607 & 6.78 & 0.618 & 5.94 & 0.09 & $0.571 \pm 0.023$ & $6.4 \pm 1.4$ & 1.01 \\
3C55 & 0.628 & 26.7 & 0.632 & 24.1 & 0.07 & $0.630 \pm 0.060$ & $9.4 \pm 1.6$ & 2.84 \\
3C103 & 0.576 & 42.8 & 0.589 & 36.5 & 0.11 & $0.617 \pm 0.099$ & $10.6 \pm 1.4$ & 4.04 \\
3C165 & 0.557 & 80.5 & 0.580 & 64.5 & 0.16 & $0.523 \pm 0.051$ & $41 \pm 5$ & 1.96 \\
3C239 & 0.673 & 2.88 & 0.678 & 2.72 & 0.04 & $0.716 \pm 0.033$ & $2.34 \pm 0.15$ & 1.23 \\
3C247 & 0.546 & 1.67 & 0.570 & 1.31 & 0.17 & $0.599 \pm 0.021$ & $1.35 \pm 0.15$ & 1.24 \\
3C280 & 0.560 & 2.61 & 0.575 & 2.22 & 0.11 & $0.694 \pm 0.031$ & $2.52 \pm 0.2$ & 1.04 \\
3C292 & 0.566 & 38.1 & 0.577 & 36.0 & 0.04 & $0.782 \pm 0.049$ & $8.4 \pm 2$ & 4.54 \\
3C294 & 0.683 & 3.65 & 0.686 & 3.48 & 0.03 & $0.898 \pm 0.067$ & $2.85 \pm 0.1$ & 1.28 \\
3C322 & 0.557 & 4.00 & 0.569 & 3.93 & 0.01 & $0.624 \pm 0.041$ & $3.65 \pm 0.3$ & 1.10 \\
3C330 & 0.543 & 6.55 & 0.563 & 5.26 & 0.15 & $0.580 \pm 0.039$ & $10.1 \pm 1.5$ & 0.65 \\
3C332 & 0.528 & 35.7 & 0.555 & 26.1 & 0.22 & $0.620 \pm 0.040$ & $23.5 \pm 4.5$ & 1.52 \\
B0908+376 & 0.514 & 19.1 & 0.543 & 12.6 & 0.29 & $0.548 \pm 0.082$ & $14 \pm 2.5$ & 1.36 \\
B1209+745 & 0.512 & 151.3 & 0.539 & 128.8 & 0.11 & $0.764 \pm 0.042$ & $81 \pm 15$ & 1.87 \\
B1312+698 & 0.506 & 112.3 & 0.536 & 88.8 & 0.17 & $0.639 \pm 0.035$ & $41 \pm 8$ & 2.74 \\
\hline
\end{tabular}

* The ratio of the standard deviation to the mean of the ages in Cols. 3 and 5 ; ** $\alpha_{\text {inj }}$ value fitted for the C.I. model using the SYNAGE package of Murgia (1996); *** the synchrotron (radiative) age of the sources compiled from the literature.

Alexander \& Leahy (1987), Leahy et al. (1989), Liu et al. (1992), and Parma et al. (1999) - are given in Col. 8 of Table 3. As shown, the synchrotron ages and our dynamical age estimates are comparable for the young sources, i.e. those whose ages are less or much less than $10 \mathrm{Myr}$. Such behavior is in fact consistent with the conclusions of Blundell \& Rawlings (2000), who argue that the spectral aging analysis is most likely erroneous for lobes older than $10 \mathrm{Myr}$. Indeed, in the case of the largest (and oldest) sources from Table 3 (3C 55, 3C 103, 3C 292, B1209+745, $\mathrm{B} 1312+698)$, the ratios of the dynamical age estimates to the synchrotron ages (see Col. 9 of Table 3 ) rise to a factor of 2-4. This also agrees with the earlier result for this ratio determined for the three giant radio galaxies: J0912+3510, J1343+3758, and J1451+3357 (Jamrozy \& Machalski 2005). The "best solution" for the age of the analyzed radio galaxies vs. their synchrotron age is plotted in Fig. 9.

\subsection{The jet power, the central core density, and other physical parameters}

Given the age determined for a given source, we can derive its other physical parameters. It was shown in Sect. 4.1 that each "age solution" determined by the intersection of the age curves in the $Q_{\text {jet }}-\rho_{0}$ diagrams (Figs. 2, 7a, and 8a) indicates unique values of the jet power $Q_{\text {jet }}$ and the central core density $\rho_{0}$. It also provides unique values of the source (cocoon) energy density and pressure, $u_{\mathrm{c}}$ and $p_{\mathrm{c}}$, respectively, as well as the total radiative energy $E_{\text {tot }}$ (see Table 1 ). We note, that the minimum total cocoon energy (which gives the "best solution" of the age) naturally corresponds to the equivalent minimum of $u_{\mathrm{c}}$ and $p_{\mathrm{c}}$.

All the physical parameters corresponding to the "best solution" of age for the sources considered in this paper, obtained with $\gamma_{\mathrm{i}, \max }=10^{7}$ and given in Table 3, are collected in Table 4. The last column of Table 4 gives, in addition, the ratio of the mean expansion velocity of the jet head to the speed of light, i.e. one half of the projected linear size of a given source divided by its age estimate.

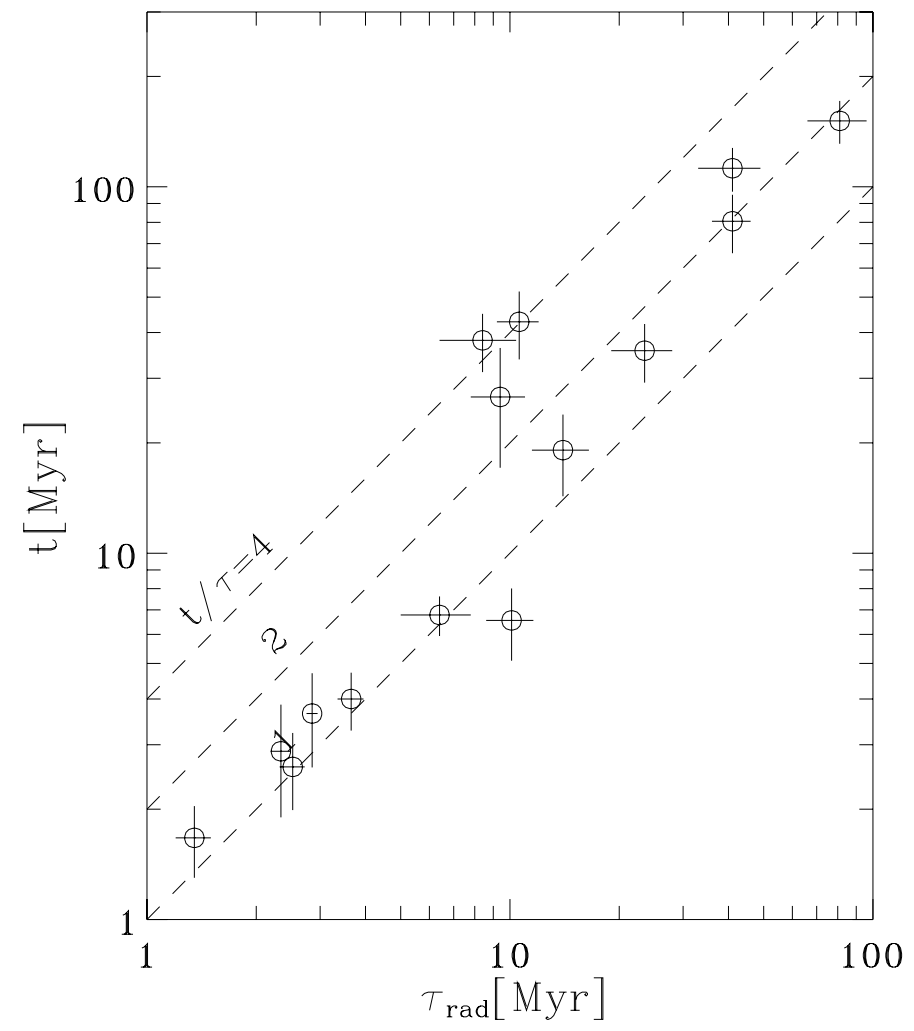

Fig. 9. "Best solution" age vs. the synchrotron age for the radio galaxies in Table 2.

\subsection{A limitation of the method}

A justified question may be raised: starting from what age/size (or equivalently stage of the source's evolution) can the described method be applied? A priori the method is applicable to the sources for which radio emission is dominated by the lobes or cocoons evolving within a power-law ambient mediumdensity profile. Therefore, our method cannot be applied directly to the young (CSO-type) sources whose linear sizes are $<1 \mathrm{kpc}$, 
Table 4. Physical parameters of the sources from Table 2.

\begin{tabular}{llllllll}
\hline \hline Source & $\log \left(Q_{\text {jet }}[\mathrm{W}]\right)$ & $\log \left(\rho_{0}\left[\mathrm{~kg} \mathrm{~m}^{-3}\right]\right)$ & $\log \left(u_{\mathrm{c}}\left[\mathrm{J} \mathrm{m}^{-3}\right]\right)$ & $\log \left(p_{\mathrm{c}}\left[\mathrm{N} \mathrm{m}^{-2}\right]\right)$ & $\log \left(E_{\text {tot }}[\mathrm{J}]\right)$ & $Q_{\text {jet }} t / E_{\text {tot }}$ & $v_{\mathrm{h}} / c$ \\
\hline Cyg A & 39.19 & -22.45 & -10.42 & -10.59 & 53.17 & 2.24 & 0.032 \\
3C 55 & 39.28 & -22.02 & -11.24 & -11.42 & 53.68 & 3.38 & 0.030 \\
3C 103 & 38.54 & -21.92 & -11.63 & -11.81 & 53.10 & 3.70 & 0.017 \\
3C 165 & 37.99 & -22.46 & -12.06 & -12.22 & 53.08 & 2.06 & 0.008 \\
3C 239 & 39.90 & -22.91 & -9.93 & -10.11 & 53.60 & 1.84 & 0.054 \\
3C 247 & 39.19 & -23.75 & -10.50 & -10.68 & 52.51 & 2.61 & 0.093 \\
3C 280 & 39.52 & -23.50 & -10.51 & -10.68 & 53.17 & 1.90 & 0.071 \\
3C 292 & 39.18 & -22.29 & -11.83 & -12.01 & 53.63 & 4.54 & 0.082 \\
3C 294 & 39.95 & -22.65 & -10.01 & -10.18 & 53.67 & 2.21 & 0.060 \\
3C 322 & 39.91 & -23.30 & -10.82 & -11.00 & 53.58 & 2.70 & 0.115 \\
3C 330 & 39.41 & -22.71 & -11.15 & -11.33 & 52.93 & 6.26 & 0.098 \\
3C 332 & 37.45 & -22.61 & -12.06 & -12.24 & 52.08 & 2.67 & 0.010 \\
B 0908+376 & 36.52 & -23.62 & -12.23 & -12.41 & 51.06 & 1.73 & 0.006 \\
B1209+745 & 36.98 & -22.80 & -13.37 & -13.54 & 52.17 & 3.07 & 0.009 \\
B1312+698 & 37.20 & -23.00 & -13.25 & -13.42 & 52.33 & 2.93 & 0.012 \\
\hline
\end{tabular}

because it is unlikely that their observed radio emission can be entirely attributed to the non-relativistic lobes. In particular, in many of these sources contribution from the underlying relativistic jets and bright mildly-relativistic hotspots to the total radiative output may be significant. Finally, it is also not obvious if some (small) fraction of CSOs is "frustrated" by the dense ambient medium, as advocated by many authors (see, e.g., De Young 1993; Alexander 2000).

Slightly larger versions of CSOs, called "medium symmetric objects" (MSOs, or "compact steep-spectrum" sources, $\mathrm{CSS}$, if the spectral characteristics are considered), are probably more promising in this context, since their projected linear sizes are typically $1-10 \mathrm{kpc}$. For example, the dynamical age estimates determined with our method for two of such objects from the sample of Murgia et al. (1999) are $142 \pm 35 \mathrm{kyr}\left(\alpha_{\text {inj }}=\right.$ $0.571 \pm 0.020)$ for $3 \mathrm{C} 67$ with $D=11 \mathrm{kpc}$ and $258 \pm 67 \mathrm{kyr}$ $\left(\alpha_{\text {inj }}=0.753 \pm 0.100\right)$ for $3 \mathrm{C} 186$ with $D=15.7 \mathrm{kpc}$. The above dynamical age estimates are larger by a factor of 2-3 than the synchrotron ages found by Murgia et al. (which are 51 and $113 \mathrm{kyr}$ for 3C 67 and 3C 186, respectively). However, such a discrepancy may be expected, as these synchrotron ages were determined using the break frequency in the spectrum of the entire source. That spectrum must be dominated by the radiation produced by the hottest regions of a given object, probably very close to a jet terminal shock. For this reason, the spectral ages found by Murgia et al. can be significantly lower than the dynamical ages derived by us. On the other hand, it is worth emphasizing that the $\alpha_{\text {inj }}$ values fitted by Murgia et al. in the frame of the C.I. model and our values determined from the minimum jet kinetic energy agree between themselves very well.

\section{Remarks on the injection electron spectrum}

Jets in powerful radio sources were considered as being characterized by sub- or eventually mildly-relativistic bulk velocities on large (hundreds of kpc) scales, $v_{\text {jet }}<0.95 c$ (e.g., Wardle \& Aarons 1997). Also, advanced velocities of their terminal features, the hotspots, were known to be non-relativistic, $v_{\text {hs }}<0.1 c$ (Scheuer 1995). For these reasons, it was widely assumed that the double-shock structures formed at the heads of the jets in quasars and FR II radio galaxies were in fact always non-relativistic phenomena. In particular, it was argued that the non-relativistic reverse shock formed at the jet terminal point was the place of the efficient diffusive (Fermi I) particle acceleration process, leading to formation of the power-law particle-energy spectrum within the hotspot, injected later into the expanding lobes/cocoons by means of the plasma backflow.

It is known that the well-established theory for particleshock acceleration in the non-relativistic test-particle limit predicts a universal power-law index of the accelerated particle continuum $p=2$, weakly dependent on the plasma conditions on both sides of the shock (e.g., Blandford \& Eichler 1987). Interestingly, radio spectral indices of powerful hotspots are indeed close to - though not always and not exactly - the corresponding value $\alpha_{\text {inj }}=(p-1) / 2=0.5$. Such an agreement supported the "non-relativistic shock" paradigm for the origin of non-thermal electrons within heads and cocoons of FR II type radio sources. It stimulated further development of this idea in the context of modeling the non-thermal hotspots' emission (Heavens \& Meisenheimer 1987), enabling the successful explanation of several observational features (e.g., Meisenheimer et al. 1989, 1997). An important implication is that, in the framework of such a "standard" model, the injection spectral index for all the cocoons of powerful radio sources is expected to be strictly $\alpha_{\text {inj }}=0.5$.

As mentioned in Sect. 2.3, a number of low-frequency radio observations of FR II sources indicate that there is no universal injection spectral index for their cocoons (see in this context Rudnick et al. 1994). This conclusion is also supported by the detailed spectral aging studies of the few brightest objects of this type, such as Cyg A (Carilli et al. 1991). In addition, the standard assumption regarding non-relativistic velocities of the large-scale jets was recently questioned by the most recent X-ray observations (e.g., Celotti et al. 2001; Tavecchio et al. 2005). If this is indeed the case, then one should expect significant modifications in the energy spectrum of the accelerated particles when compared to the non-relativistic limit. Moreover, as argued below, the assumption regarding a single power-law form of the injected electrons is also most likely far from being realistic. For these reasons, in our analysis we decided to treat $\alpha_{\text {inj }}$ as a basically free "effective" parameter, which may be different for different sources and which may be far from the canonical value of 0.5 .

\subsection{Low-energy electrons}

In this paper we assumed that the power-law continuum of electrons injected by the hotspot to the cocoons extends down to electron Lorentz factors $\gamma_{\mathrm{i} \text {,min }}=1$. In general, synchrotron emission of the electrons with $\gamma_{\mathrm{i}}<10^{3}$ cannot be observed directly 
from the hotspots, since it corresponds to frequencies $<100 \mathrm{MHz}$ for a typical value of the hotspots' magnetic field $\sim 100 \mu \mathrm{G}$. Thus, the assumption regarding the low-energy electron spectrum cannot be verified by observations in a simple way and should rather be constrained by the theory of particle acceleration.

Unfortunately, such a theory is not yet well developed. We note in this context that powerful jets of large scales are most likely dynamically dominated by cold protons (see, e.g., Sikora et al. 2005, and references therein). This means that the thickness of the terminal reverse shock should be roughly a few gyroradii of the non- or mildly-relativistic protons, such that the energies of the electrons that are able to undergo diffusive shock acceleration must already be ultrarelativistic, $\gamma_{\mathrm{i}}>m_{\mathrm{p}} / m_{\mathrm{e}}$. The questions are then (i) which process is responsible for acceleration of $1<\gamma_{i}<10^{3}-10^{4}$ electrons; (ii) if the resulting low-energy part of the electron continuum is characterized by a power-law form at all; and (iii) if so, whether the low-energy spectral index is the same as for the high-energy part of the electron continuum $\left(\gamma_{\mathrm{i}}>10^{3}-10^{4}\right)$, originating (by assumption) from the diffusive shock acceleration process.

Such problems in the context of FR II hotspots were not discussed much in the literature, with only a few papers addressing these important issues. For example, Kino \& Takahara (2004) analyze limiting possibilities for the efficient and non-efficient temperature coupling between dynamically dominating protons and electrons at the jet terminal shock, resulting in the formation of an ultrarelativistic Maxwellian-type electron distribution at energies $\gamma_{\mathrm{i}} \sim \Gamma_{\text {jet }} m_{\mathrm{p}} / m_{\mathrm{e}}$ and $\gamma_{\mathrm{i}} \sim \Gamma_{\text {jet }}$, respectively (where $\Gamma_{\text {jet }}$ is the jet bulk Lorentz factor), followed by the shock-formed highenergy power-law tail. They argue that multiwavelength observations of the Cyg A hotspots contradict both possibilities. This implies either an electron-positron jet composition (as preferred by Kino \& Takahara 2004) or simply more complex (broadband) energy spectrum of low-energy electrons (instead of an almost monoenergetical Maxwellian distribution). In fact, formation of such a broad-band energy spectrum seems to be more likely, since any energy/temperature coupling between protons and electrons cannot be collisional in nature in the case of extragalactic jets, but must be mediated by the magnetic field and plasma turbulence (see in this context Hoshino et al. 1992).

To sum up, if the dynamical dominance of the cold protons within powerful extragalactic jets is the case, one should expect (possibly significant) differences in the spectral shape of the low-energy $\left(1<\gamma_{\mathrm{i}}<10^{3}-10^{4}\right)$ and high-energy $\left(\gamma_{\mathrm{i}}>10^{4}\right)$ parts of the electron continuum formed at jet terminal shocks. Indeed, low-frequency flattening observed in the radio emission of Cyg A hotspots (Carilli et al. 1991), as well as the most recent analysis of the X-ray emission of the lobes of giant radio galaxy 4C 39.24 (Blundell et al. 2006), are both consistent with such an expectation. This in turn implies that the broad-band electron spectrum injected by the hotspots to the expanding cocoons in reality does not have a simple, single power-law form.

\subsection{Relativistic shock velocities}

In the section above we have argued that the low-energy part of the electron distribution formed at the hotspots of powerful jets is probably broad-band, possibly even power-law, but with the (unconstrained at the moment) power-law slope most likely different from the one characterizing its high-energy part. The difference may be due to the fact that, while the low-energy part is formed by poorly known processes of energy exchange between different plasma species within the collisionless shock front, the high-energy part is supposed to originate in the "standard" Fermi I acceleration mechanism. Yet another issue is whether this high-energy part always possesses some universal value of its power-law slope, the same for every powerful radio galaxy. We believe not since, even if the diffusive shock acceleration process is indeed responsible for formation of the latter, the expected relativistic effects will modify the resulting electron spectrum when compared to the non-relativistic test-particle model value $p=2$.

As already mentioned, there is growing evidence that relativistic velocities are present in large-scale FR II and quasar jets, so that the reverse shocks formed at the jet heads are at least mildly relativistic. An important fact in this context is that polarization studies usually indicate magnetic fields perpendicular to the jet axis within the hotspots (even in the case of giant-sized radio galaxies, see Machalski et al. 2006), leading to an oblique orientation of the mean magnetic field lines with respect to the reverse shock front. It was noted by Begelman \& Kirk (1990) that oblique relativistic shocks are likely to be superluminal, in the sense that the shock velocity projected on the magnetic field vector exceeds the speed of light. In such a situation, a diffusive (Fermi I) acceleration process may not be possible at all, since particles moving on average along the magnetic field lines are not able to cross the shock front more than once. Moreover, as shown recently by Niemiec \& Ostrowski (2004), even if the magnetic field configuration is luckily such that the diffusive particle scattering on both sides of the (mildly-)relativistic shock is in principle possible, the resulting energy spectrum of the accelerated particles strongly depends on the turbulence conditions in vicinity of the shock. As a result, a variety of spectral shapes for the accelerated particles are obtained, including different spectral indices and deviations from power-law forms. We believe that this may be partly the reason for the different values of the injection spectral index obtained here for different sources.

\subsection{Jet/hotspot intermittency}

Multiple hotspots detected on the same sides of many FR II radio galaxies and quasars indicate that the jet terminal features are intermittent in nature (see, e.g., Scheuer 1982; Williams \& Gull 1985; Leahy \& Perley 1995). Typically, a pair of the hotspots is observed on one side of the cocoon, with one feature being stronger and more extended ("secondary hotspot"), and the other one weaker and more compact ("primary hotspot"), as in the famous source Cyg A. The secondary and primary hotspots are identified with "older (extinguishing)" and "younger (recent)" jet termination points. We note that such an intermittency may be a direct consequence of the intermittent nature of the jet phenomenon, discussed in the literature for a number of reasons (e.g., Reynolds \& Begelman 1997; Stawarz et al. 2004).

Whatever the case is, the issue of interest here is that multiple hotspots differ in their multifrequency radiative and geometrical properties (see Hardcastle et al. 1998). This should in fact be expected as a natural consequence of the aging/evolutionary processes taking place in a mildly-relativistic shock front "fed" by intermittent jet outflow. An important consequence of this fact is that the electron energy distribution injected into the cocoons by the hotspots, characterized by much shorter lifetimes than the lifetime of the whole radio source, is in fact an average over different activity phases of the jet's terminal features. As a result, the injection spectral index inferred from analyzing of the cocoon's radio continuum may differ substantially from the low-frequency spectral index of the currently active hotspot in a given radio source, as suggested in our modeling. 
The above discussion, pointing out all the reasons one should not expect a single broad-band power-law shape of the electrons accelerated at the jet head and injected into the expanding cocoons agrees with the results of detailed investigation of radio continuum in FR II sources by, e.g., Rudnick et al. (1994), Treichel et al. (2001), and Young et al. (2005). By applying sophisticated technique of radio data analysis, these authors found that there is no universal injection power-law spectral index for the cocoons of powerful radio sources, that the injected population of relativistic electrons thereby is characterized by complex ("curved") spectral shape, and that a variety of distinct spectral features exist within the extended lobes. We argue, however, that for the purpose of simple estimates of the sources' main parameters (such as ages, jet powers, etc.) as presented here, a single power-law injection spectrum may well be a relatively good approximation. The effective spectral index of such a model particle energy distribution may, however, be different for different sources. In this context we note that, somewhat surprisingly, our analysis suggests a relatively narrow range for this parameter, namely $p=2.0-2.4$.

\section{Conclusions}

Here we propose a new approach to determining the ages of powerful radio sources, on the one hand, by exploiting the dynamical model developed for these objects by Kaiser et al. (1997; KDA), and on the other hand, using multifrequency radio observations not necessarily restricted to the high-resolution ones. The KDA model is chosen for its minimal number of free parameters when compared to the more sophisticated scenarios by Blundell et al. (1999) or Manolakou \& Kirk (2002).

We applied the assumed dynamical model to a number of FR II type radio galaxies observed at different radio frequencies, and fit - for each frequency separately - the model-free parameters to the sources' observed quantities. Such a procedure, enlarging in fact a number of observables, enables us to relatively precisely determine ages and other crucial characteristics (such as the jet kinetic power) of the analyzed sources. We show that the resulting age estimates extend from about $1 \mathrm{Myr}$ for the small but powerful high-redshift radio galaxies to over $100 \mathrm{Myr}$ for the giant radio galaxies. The estimated ages agree very well with the ones obtained by means of the "classical" spectral aging method for objects not older than $10 \mathrm{Myr}$, for which good-quality data are available. Meanwhile, in the case of sources older than $10 \mathrm{Myr}$, our age estimates are systematically higher (by a factor of 2-4) than the ages obtained by means of spectral aging analysis. Such behavior is in fact consistent with the conclusions of Blundell \& Rawlings (2000), who argue that the spectral aging analysis is most likely erroneous for the lobes older than 10 Myr.

Interestingly, the ages of FRII-type sources estimated by using this method always correspond to "realistic" values of the jetadvance velocity $\leq 0.3 c$, i.e. consistent with those measured directly with the VLBI technique in many of the CSO-type sources (see Sect. 5.2). Thus, we believe that the method presented here is also applicable in the case of the oldest radio galaxies and/or ones for which the only available low-resolution radio data do not allow for detailed spectral aging studies (i.e., for majority of powerful radio sources). This implies that the applied procedure of favoring the value of the injection spectral index that corresponds to the minimum of the cocoons' total energy (see Sect. 4.3), although to some level arbitrary, nevertheless gives very reasonable results.
Our analysis indicates that the main factor precluding precise age determination for FR II type radio galaxies regards the poorly known shape of the initial electron energy distribution injected by the jet terminal shocks into the expanding lobes/cocoons. We considered this issue briefly and conclude that the broad-band single power-law form assumed here (and in all the other analogous models) may be accurate enough for the presented estimates, although it most likely does not strictly correspond to some well-defined realistic particle acceleration process. Instead, it should be considered as a simplest model approximation of the initial electron continuum, averaged over a very broad energy range and over the age of the source. The effective spectral index of such a distribution may be different for different sources, however within the relatively narrow range $p=2.0-2.4$ suggested by our modeling.

In a subsequent paper we intend to apply this method of age determination in a large sample of FR II-type (but also younger, MSO-type) radio sources.

Acknowledgements. This project was supported by MEiN with funding for scientific research in the years 2005-2007 under contract No. 0425/P03/2005/29. Ł.S. also acknowledges support by the ENIGMA Network through grant HPRNCT-2002-00321 and by MEiN through the grant 1-P03D-003-29 in the years 2005-2008.

\section{References}

Alexander, P. 2000, MNRAS, 319, 8

Alexander, P., \& Leahy, J. P. 1987, MNRAS, 225, 1

Barai, P., \& Wiita, P. J. 2006, MNRAS, 372, 381

Barthel, P. D. 1989, ApJ, 336, 606

Begelman, M. C., \& Cioffi, D. F. 1989, ApJ, 345, L21

Begelman, M. C., \& Kirk, J. G. 1990, ApJ, 353, 66

Blandford, R. D., \& Rees, M. J. 1974, MNRAS, 169, 395

Blandford, R. D., \& Eichler, D. 1987, Phys. Rep., 154, 1

Blundell, K. L., \& Rawlings, S. 2000, AJ, 119, 1111

Blundell, K. M., Rawlings, S., \& Willott, C. J. 1999, AJ, 117, 677

Blundell, K. M., Fabian, A. C., Crawford, C. S., Erlund, M. C., \& Celotti, A. 2006, ApJ, 644, 13

Canizares, C. R., Fabbiano, G., \& Trincheiri, G. 1987, ApJ, 312, 503

Carilli, C. L., Perley, R. A., Dreher, J. W., \& Leahy, J. P. 1991, ApJ, 383, 554

Celotti, A., Ghisellini, G., \& Chiaberge, M. 2001, MNRAS, 321, L1

Chyży, K. T. 1997, MNRAS, 289, 355

Croston, J. H., Hardcastle, M. J., Harris, D. E., et al. 2005, ApJ, 626, 733

Daly, R. A. 1995, ApJ. 454, 580

De Young, D. S. 1993, ApJ, 402, 95

Eilek, J. A., \& Arendt, P. N. 1996, ApJ, 457, 150

Falle, S. A. E. G. 1991, MNRAS, 250, 581

Gopal-Krishna 1988, A\&A, 192, 37

Gopal-Krishna, \& Wiita, P. J. 1987, MNRAS, 226, 531

Gugliucci, N. E., Taylor, G. B., Peck, A. B., \& Giroletti, M., ApJ, 622, 136

Hardcastle, M. J., Alexander, P., Pooley, G. G., \& Riley, J. M. 1998, MNRAS, 296, 445

Hardcastle, M. J., Harris, D. E., Worrall, D. M., \& Birkinshaw, M. 2004, ApJ, 612,729

Heavens, A. F., \& Meisenheimer, K. 1987, MNRAS, 225, 335

Hoshino, M., Arons, J., Gallant, Y. A., \& Langdon, A. B. 1992, ApJ, 390, 454

Jamrozy, M., \& Machalski, J. 2005, Baltic Astr., 14, 381

Jones, T. W., Ryu, D., \& Engel, A. 1999, ApJ, 512, 105

Kaiser, C. R. 2000, A\&A, 362, 447 (K2000)

Kaiser, C. R., \& Alexander, P. 1997, MNRAS, 286, 215 (KA)

Kaiser, C. R., Dennett-Thorpe, J., \& Alexander, P. 1997, MNRAS, 292, 723 (KDA)

Kataoka, J., \& Stawarz, Ł. 2005, ApJ, 622, 797

Katz-Stone, D. M., \& Rudnick, L. 1997, ApJ, 479, 258

Kawakatu, N., \& Kino, M. 2006, MNRAS, 370, 1513

King, I. R. 1972, ApJ, 174, L123

Kino M., \& Takahara, F. 2004, MNRAS, 349, 336

Kino, M., \& Kawakatu, N. 2005, MNRAS, 364, 659

Klein, U., Mack, K.-H., Gregorini, L., \& Parma, P. 1995, A\&A, 303, 427

Laing, R. A., \& Peacock, J. A. 1980, MNRAS, 190, 903

Leahy J. P., \& Perley, R. A. 1995, MNRAS, 277, 1097

Leahy, J. P., Muxlow, T. W. B., \& Stephens, P. W. 1989, MNRAS, 239, 401 
Liu, R, Pooley, G., \& Riley, J. M. 1992, MNRAS, 257, 545

Machalski, J., Chyży, K. T., \& Jamrozy, M. 2004a, AcA, 54, 249

Machalski, J., Chyży, K. T., \& Jamrozy, M. 2004b, AcA, 54, 391

Machalski, J., Jamrozy, M., Zoła, S., \& Kozieł, D. 2006, A\&A, 454, 85

Macklin, J. T. 1982, MNRAS, 199, 1119

Manolakou, K., \& Kirk, J. G. 2002, A\&A, 391, 127

Meisenheimer K., Röser, H.-J., Hiltner, P. R., et al. 1989, A\&A, 219, 63

Meisenheimer, K., Yates, M. G., \& Röser, H.-J. 1997, A\&A, 325, 57

Murgia, M. 1996, Laurea Thesis, University of Bologna

Murgia, M., Fanti, C., Fanti, R., et al. 1999, A\&A, 345, 769

Myers, S. T., \& Spangler, S. R. 1985, ApJ, 291, 52

Nath, B. B. 1995, MNRAS, 274, 208

Niemiec, J., \& Ostrowski, M. 2004, ApJ, 610, 851

O'Dea, C. P. 1998, PASP, 110, 493

Owsianik, I., \& Conway, J. E. 1998, A\&A, 337, 69

Parma, P., Murgia, M., Morganti, R., et al. 1999, A\&A, 344, 7

Polatidis, A. G., \& Conway, J. E. 2003, PASA, 20, 69

Reynolds, C. S., \& Begelman, M. C. 1997, ApJ, 487, 135
Rudnick, L., Katz-Stone, D., \& Anderson, M. 1994, ApJS, 90, 955

Scheuer, P. A. G. 1974, MNRAS, 166, 513

Scheuer P. A. G. 1982, in Extragalactic radio sources (D. Reidel Publishing Co), IAU Symp., 97, 163

Scheuer, P. A. G. 1995, MNRAS, 277, 331

Sikora, M., Begelman, M. C., Madejski, G. M., \& Lasota, J.-P. 2005, ApJ, 625, 72

Stawarz, Ł., Sikora, M., Ostrowski, M., \& Begelman, M. C. 2004, ApJ, 608, 95 Tavecchio, F., Cerutti, R., Maraschi, L., et al. 2005, ApJ, 630, 721

Treichel, K., Rudnick, L., Hardcastle, M. J., \& Leahy, J. P. 2001, ApJ, 561, 691 van Breugel, W., \& McCarthy, P. J. 1990, in Evolution of galaxies, ed. R. G. Kron, ASP Conf. Ser., 10, 359

Wardle, J. F. C., \& Aaron, S. E. 1997, MNRAS, 286, 425

Wellman, G. F., Daly, R. A., \& Wan, L. 1997, ApJ, 480, 96

Williams, A. G., \& Gull, S. F. 1985, Nature, 313, 34

Willott, C. J., Rawlings, S., Blundell, K. M., \& Lacy, M. 1999, MNRAS, 309, 101

Young, A., Rudnick, L., Katz, D., et al. 2005, ApJ, 626, 748 